\title{
Endoscopy during neurotomy of the nervus intermedius for nervus intermedius neuralgia: a case report
}

\author{
Zhuhuan Song ${ }^{1}$, Jian Chen ${ }^{1}$, Jianhong Shen ${ }^{1}$, Zhongzheng Jia ${ }^{2}$, Qinwei Wang ${ }^{1}$, Shichen Jiang ${ }^{1}$, Xide Xu $^{1}$, \\ Wei Shi ${ }^{1}$ \\ ${ }^{1}$ Department of Neurosurgery, Jiangsu Clinical Medicine Center of Tissue Engineering, Nerve Injury Repair and the Training Base of \\ Neuroendoscopic Physician under Chinese Medical Doctor Association, Affiliated Hospital of Nantong University, Nantong, China; ${ }^{2}$ Department of \\ Medical Imaging, Affiliated Hospital of Nantong University, Nantong, China \\ Correspondence to: Wei Shi, Xide Xu. Department of Neurosurgery, Jiangsu Clinical Medicine Center of Tissue Engineering, Nerve Injury Repair and \\ the Training Base of Neuroendoscopic Physician under Chinese Medical Doctor Association, Affiliated Hospital of Nantong University, 20 Xi Si \\ Road, Chongchuan District, Nantong 226001, China. Email: fysw@ntu.edu.cn; xidexu@163.com.
}

\begin{abstract}
Nervus intermedius neuralgia (NIN) is a rare craniofacial neuralgia with features of paroxysmal pain in the deep ear. Because of sensory nerves overlap in the ear, the diagnosis of NIN is often difficult and not definitive. Here, we present the case of a 70-year-old woman who had deep-ear pain for more than 4 years and was diagnosed with trigeminal neuralgia and treated with carbamazepine without relief in another hospital. Magnetic resonance tomographic angiography revealed no neurovascular conflict with the trigeminal nerve, whereas the anterior inferior cerebellar artery (AICA) was close to the VII/VIII complex. We performed left-sided suboccipital retrosigmoid craniotomy. Surgical exploration under endoscopy clearly showed that the nervus intermedius was compressed by the AICA from behind. The ear pain was completely relieved immediately after nervus intermedius sectioning. The intraoperative findings and postoperative results confirmed that the compression of the nervus intermedius by the AICA caused the otalgia. A patient's specific pain, combined with preoperative imaging examination, is useful in the diagnosis of NIN. Neuroendoscopy has the advantages of enabling a clear field of view and close observation, thus aiding in the identification and accurate cutting of the nervus intermedius during the operation.
\end{abstract}

Keywords: Nervus intermedius neuralgia (NIN); otalgia; endoscopy; magnetic resonance tomographic angiography

Submitted Aug 18, 2020. Accepted for publication Nov 08, 2020.

doi: 10.21037/atm-20-5951

View this article at: http://dx.doi.org/10.21037/atm-20-5951

\section{Introduction}

Nervus intermedius neuralgia (NIN), also known as facial neuralgia or geniculate neuralgia, has the distinctive features of intermittent pain in the deep ear, which lasts for seconds or minutes, and is sometimes accompanied by lacrimation, and taste and hearing changes. Given the complexity of sensory innervation in the ear (the 5 th, 7 th, 9th or 10th cranial nerves, and the $2 \mathrm{nd}$ and 3 rd cervical nerves) (1), both the diagnosis and treatment of NIN are challenging. Since 1932, only approximately 150 cases of NIN have been reported (2). The number of cases is far smaller than those of trigeminal neuralgia and glossopharyngeal neuralgia. In this report, we treated a patient with pain in the external auditory canals, whose symptoms all disappeared after nervus intermedius sectioning under endoscopy, thus further confirming the diagnosis of NIN. This report discusses the clinical features, differential diagnosis, imaging analysis and endoscopic findings of this case. We present the following article in accordance with the CARE reporting checklist (available at http://dx.doi.org/10.21037/atm-20-5951). 


\section{Case presentation}

A 70-year-old woman presented with a 4-year history of ear pain on the left side, covering the deep part of the ear, the front of the ear screen and the auricle. The pain was paroxysmal, and needle-like; lasted for seconds or even minutes without regular intervals; and was accompanied by lacrimation in the left eye. After being diagnosed with trigeminal neuralgia in many other clinics, the patient was treated with carbamazepine, which had no clear effect. She presented to the Department of Neurosurgery of the Affiliated Hospital of Nantong University in June of 2020.

The patient had experienced hypertension for more than 10 years and received oral reserpine. She denied diabetes, similar diseases, infectious diseases, genetic diseases, psychosocial diseases and tumor diseases. We performed magnetic resonance tomographic angiography. No tumor was found in the left cerebellopontine angle, and no vessels were found throughout the trigeminal nerve (Figure 1A,B,C). There were suspected vessels nearing the surfaces of the medial glossopharyngeal nerves (Figure 1D), and the AICA was close to the VII/VIII complex (Figure 1E,F,G). On the basis of the clinical symptoms and photographic results, we considered NIN, but the possibility of trigeminal neuralgia and glossopharyngeal neuralgia could not be ruled out. We decided to explore the CNV, CNIX and VII/VIII complexes under endoscopy 7 days after stopping the reserpine.

During the surgery, we performed a suboccipital retrosigmoid craniotomy on the left, made an incision as long as $5 \mathrm{~cm}$ in the skin behind the ear, and exposed the sigmoid sinus and transverse sinus. After we opened dura mater and discharged the cerebrospinal fluid, we observed no vessels throughout the trigeminal nerve under endoscopy (Figure 2A) (neuroendoscope, Image 1s, Storz, Germany). The glossopharyngeal and vagus nerves showed no vascular compression (Figure 2B,C). We explored the VII/VIII complex by endoscopy and observed that the nervus intermedius was clearly located between the vestibulocochlear nerve and the facial nerve, was composed of multiple nerve root filaments and was closely associated with the AICA (Figure 2D). On the basis of the clinical presentation, imaging data and endoscopic findings, the patient was diagnosed with NIN and treated with nervus intermedius section (Figure 2E). The operation proceeded smoothly and there was no neurovascular damage (Figure $2 F$ ). No drainage tube was placed during the operation, and there was no need to remove the suture after the operation, owing to the use of absorbable sutures. The patient's ear pain was completely relieved immediately after the surgery, thus further confirming the diagnosis of NIN. Three months after the surgery, the patient had no ear pain, dry eyes, abnormal taste, hearing loss, facial paralysis or other neurological deficits. The patient and family members are satisfied with the operation. Follow-up observation will be continued. All procedures performed in studies involving human participants were in accordance with the ethical standards of the institutional and national research committee(s) and with the Helsinki Declaration (as revised in 2013).

\section{Timeline}

2010: Hypertension was found, and reserpine was taken orally regularly for symptomatic treatment. 2016: Left ear pain was found. 2018: Trigeminal neuralgia was diagnosed in another hospital, and carbamazepine was taken orally. At first, the pain was relieved with two tablets/day; the dose was later gradually increased to four tablets/day, but the pain was not significantly eased. May 4, 2020: The patient went to the outpatient clinic of Neurosurgery Department of Affiliated Hospital of Nantong University for MRTA examination, which indicated that the AICA was close to the VII/VIII complex. June 22, 2020: The patient was admitted to the Neurosurgery Department of Affiliated Hospital of Nantong University. June 29, 2020: 7 days after the withdrawal of reserpine, the nervus intermedius was severed under neuroendoscopy, and the operation was successful. The patient's pain disappeared immediately after surgery, without neurological deficits. July 6, 2020: The patient recovered well, and was discharged from hospital and followed up.

\section{Discussion}

The nervus intermedius involves sensory and parasympathetic nerve fibers, and it dominates the lacrimal gland, the glands of the nose and palate, as well as the submandibular, sublingual and accessory salivary glands. The sensation of taste in two-thirds of the anterior tongue, and the general sensation in the area around the concha of the external ear and the external auditory canal are also controlled by the nervus intermedius (3). NIN is characterized by pain paroxysms felt in the depth of the ear, lasting for several seconds or minutes, and exhibiting intermittent occurrence; the pain is often accompanied by ipsilateral nasal mucosal 

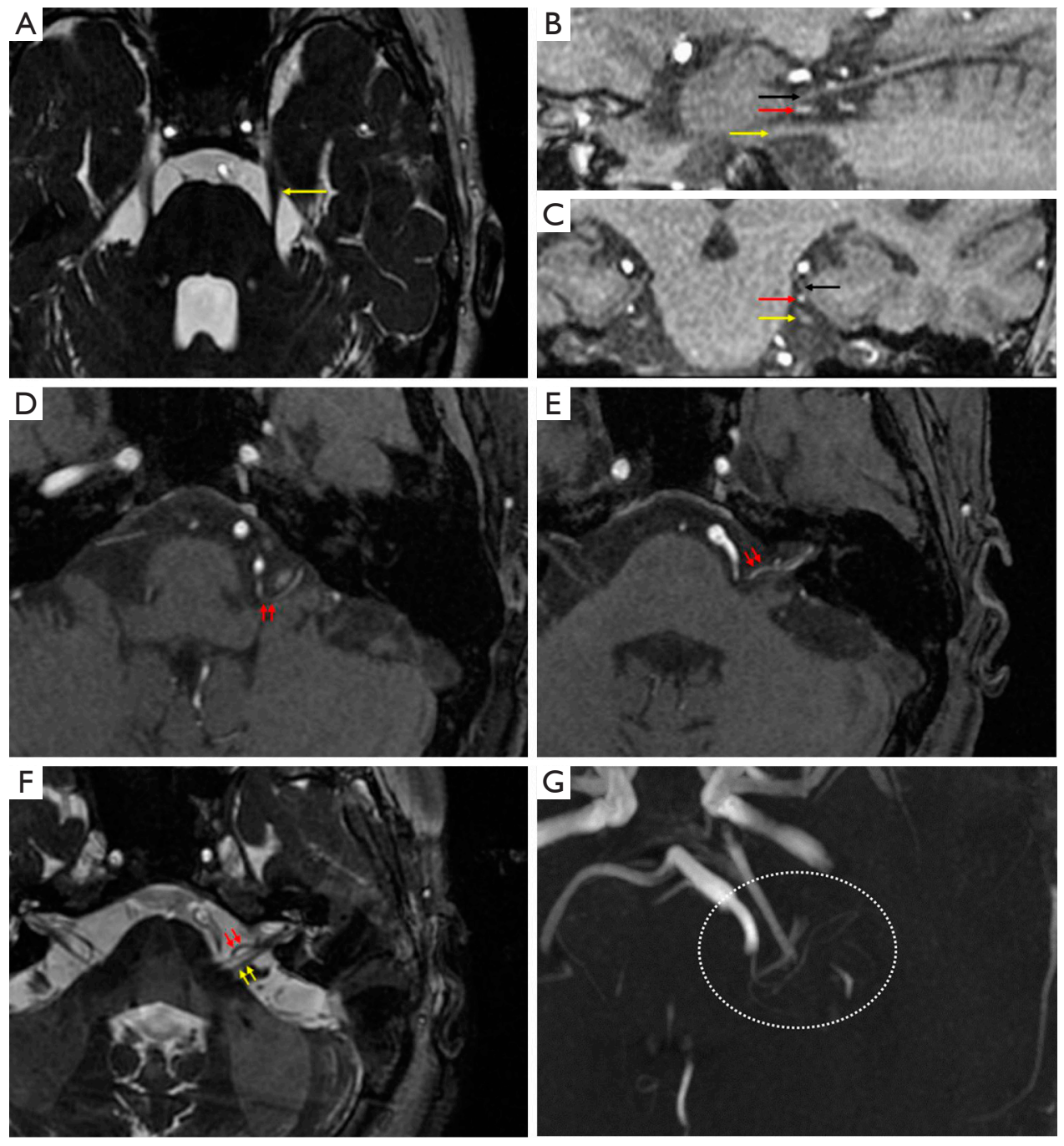

Figure 1 Preoperative magnetic resonance tomographic angiography. (A) The FIEST sequence of the axial MRTA indicates no vessels abutting the trigeminal nerve (yellow arrowhead). (B,C) The TOF sequence of the sagittal and coronal MRTA suggests that the superior cerebellar artery (red arrowhead) is located under the tentorium cerebelli (white arrowhead) and above the trigeminal nerve (yellow arrowhead), making no contact with the trigeminal nerve. (D) The TOF sequence of the axial MRTA suggests that the anterior inferior cerebellar artery (red arrowheads) is looped under the VII/VIII complex and the medial glossopharyngeal nerves. (E) The TOF sequence, (F) FIEST sequence and $(\mathrm{G})$ vascular reconstruction sequence together suggest that the loop of AICA (red arrowheads) originates from the medial glossopharyngeal nerve running along the VII/VIII complex (yellow arrowheads) to the internal auditory canal, and it turns back in the shape of an " 8 ", which is closely associated with the VII/VIII complex.

congestion and tears; changes in taste and hearing may also be present (4). In our case, the paroxysmal pain was located in the deep left ear and spread to the auricle. In addition, the patient's left eye sometimes had lacrimation, thus providing a strong basis for a diagnosis of NIN. Paroxysmal otalgia did not occur during swallowing, talking, or washing the face. All these symptoms contributed to distinguishing
NIN from trigeminal neuralgia and glossopharyngeal neuralgia.

The preoperative imaging evaluation of hemifacial spasms and trigeminal neuralgia has been improved, as evidenced by numerous reports on the preoperative imaging evaluation of cranial nerve diseases. However, NIN, with its distinct syndromes of neurovascular conflict (5), 

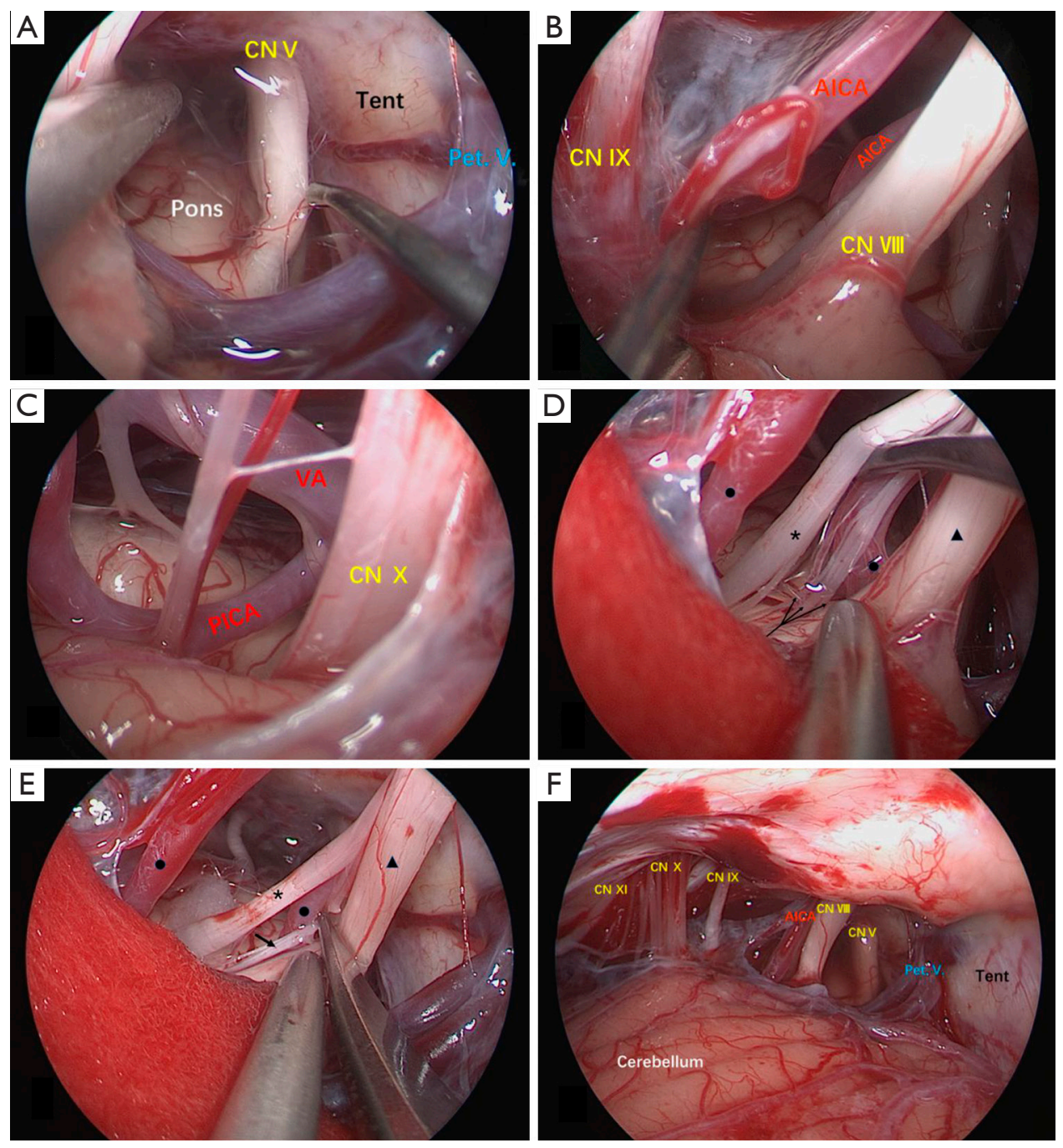

Figure 2 Intraoperative views through endoscopy. (A) No offending vessels were found around the trigeminal nerve. (B) No clear compression of the glossopharyngeal nerve by anterior inferior cerebellar artery (AICA) was observed. (C) Posterior inferior cerebellar artery (PICA) issued from the vertebral artery, and no compression of the vagus nerve by PICA or the vertebral artery was observed. (D,E) The intermediate nerve (arrowheads) was clearly visible after the facial nerve was gently lifted. Several tiny branches were observed around the trunk, and they originated from the root of the auditory nerve (triangle) near the brainstem, and the distal segments joined the facial nerve trunk (asterisk). The AICA (dot) compressed the facial nerve on the medial side. The location of compression exhibited attenuation, and neurotomy was conducted. (F) After the operation, the entire view of the posterior fossa was exposed through endoscopy, and all nerves and vessels were clearly visible.

is different: because the nervus intermedius is very thin, detecting neurovascular compression through MRI is essentially impossible. Although Burmeister et al. (6) have reported anatomical evaluation of the nervus intermedius with 3 Tesla MRI, this imaging evaluation is more difficult to perform than that for hemifacial spasm and trigeminal neuralgia. The only radiological finding of neurovascular conflict with the nervus intermedius is that some vessels relocate to the VII/VIII complex. However, for an NIN diagnosis to be made, clinical symptoms are required.

In previous reports, the AICA has been found to be the responsible vessel for NIN in most cases, and it always passes between the vestibulocochlear nerve and the facial nerve. However, in this case, the AICA compressed the 
intermediate nerves from the ventral side of the VII/VIII complex, thus causing difficulties in identifying NIN caused by neurovascular conflict. Taking advantage of the clear field of view and close observation afforded by endoscopy, we clearly observed the compression of the nervus intermedius and thus made a diagnosis of NIN.

In this case, we initially diagnosed the patient with NIN because of the typical clinical symptoms and the imaging of the compression on the ventral side of VII/VIII complex by the AICA; therefore, we decided to perform a craniotomy. The CNV, CNIX and VII/VIII complexes were explored by taking advantage of high the definition and proximate observation afforded by neuroendoscopy. Under endoscopy, we first confirmed the absence of invasion of vessels around CNV and CNIX, then made a definite diagnosis of NIN after observing the VII/VIII complex and verifying the compression of the nervus intermedius by the AICA. After experiencing pain for years and serious effects on her quality of life, the patient had a strong and urgent need for surgery to relieve the pain but was unwilling to undergo another operation because of the possibility of recurrence. After thorough discussion, we performed a nervus intermedius transection to achieve the goal of radical cure after the patient provided preemptive informed consent.

For NIN surgery, we were unable to compare the efficacy of endoscopy and microscopy, because research involving many cases would be necessary. In as much as $20 \%$ of cases, identifying the nervus intermedius from the branches of the facial nerve in the cisternal part is difficult (7). However, in this case, the clear field of view afforded by endoscopy and close observation helped us confirm the compression of the nervus intermedius by the AICA, clearly exposing the small nervus intermedius (including its branches), and minimizing reducing stretching of important structures such as the facial nerve and the auditory artery. The clear observation of compression and thinning of the nervus intermedius under endoscopy confirmed the diagnosis of NIN and also allowed us to explore all the neurovascular structures including the trigeminal nerve and glossopharyngeal vagus nerves.

Severing the nervus intermedius may result in dysfunction such as crocodile tears, xerophthalmia and allotriogeusia. Most deficits are temporary, and even the permanent adverse effects are tolerable (8). In the literature, intermedius transection has been described as an option when the nervus intermedius limits adequate mobilization of the AICA in microvascular decompression surgery for hemifacial spasms (9). In this case, there was no functional nerve injury after nervus intermedius transection, thus indicating that transection is acceptable, and permanent pain relief can be achieved simultaneously.

Vascular compression is only one of the causes of NIN, and it is only theoretical and has not been confirmed by basic research; moreover, whether simple decompression is effective has also not been confirmed in a double-blind controlled study. Because there are no reports of many cases of simple decompression, whether vascular compression can lead to NIN is controversial. Although blood pressure causing nerve pinching is clearly seen under endoscopy, severing the intermediate nerve is an effective way to address non-vascular factors, such as herpetic infection or neuroborreliosis $(10,11)$.

Although NIN occurs rarely, we should consider it in differential diagnosis for patients with symptoms of otalgia. Preoperative imaging examination of MRTA plays an important role in diagnosis and treatment. The highly clear field of view and close observation afforded by endoscopy contribute to the safety and efficacy of the surgery, and endoscopic neurosurgery shows favorable prospects for clinical application. Because this article involved only one case, observations from accurate surgical outcomes of more cases and long-term follow-up are needed.

\section{Acknowledgments}

We thank International Science Editing for editing this manuscript.

Funding: This work was supported by the Application Research Project of Nantong City (grant No. HS2017001), and the Innovation Team of the Affiliated Hospital of Nantong University.

\section{Footnote}

Reporting Checklist: The authors have completed the CARE reporting checklist. Available at http://dx.doi.org/10.21037/ atm-20-5951

Conflicts of Interest: All authors have completed the ICMJE uniform disclosure form (available at http://dx.doi. org/10.21037/atm-20-5951). The authors have no conflicts of interest to declare.

Etbical Statement: The authors are accountable for all aspects of the work in ensuring that questions associated with the accuracy or integrity of any part of the work are 
appropriately investigated and resolved. All procedures performed in studies involving human participants were in accordance with the ethical standards of the institutional and national research committee(s) and with the Helsinki Declaration (as revised in 2013). Written informed consent was obtained from the patient.

Open Access Statement: This is an Open Access article distributed in accordance with the Creative Commons Attribution-NonCommercial-NoDerivs 4.0 International License (CC BY-NC-ND 4.0), which permits the noncommercial replication and distribution of the article with the strict proviso that no changes or edits are made and the original work is properly cited (including links to both the formal publication through the relevant DOI and the license). See: https://creativecommons.org/licenses/by-nc-nd/4.0/.

\section{References}

1. Fernandes EGL, van Doormaal T, de Ru S, et al. Microvascular decompression of the VII/VIII cranial nerve complex for the treatment of intermediate nerve neuralgia: a retrospective case series. Oper Neurosurg (Hagerstown) 2018;15:378-85.

2. Tang IP, Freeman SR, Kontorinis G, et al. Geniculate neuralgia: a systematic review. J Laryngol Otol 2014;128:394-9.

3. Tubbs RS, Steck DT, Mortazavi MM, et al. The nervus intermedius: a review of its anatomy, function, pathology,

Cite this article as: Song Z, Chen J, Shen J, Jia Z, Wang Q, Jiang S, Xu X, Shi W. Endoscopy during neurotomy of the nervus intermedius for nervus intermedius neuralgia: a case report. Ann Transl Med 2021;9(2):179. doi: 10.21037/atm-205951 and role in neurosurgery. World Neurosurg 2013;79:763-7.

4. Inoue T, Shima A, Hirai H, et al. Nervus intermedius neuralgia treated with microvascular decompression: a case report and review of the literature. NMC Case Rep J 2017;4:75-8.

5. Sakas DE, Panourias IG, Stranjalis G, et al. Paroxysmal otalgia due to compression of the intermediate nerve: a distinct syndrome of neurovascular conflict confirmed by neuroimaging. Case report. J Neurosurg 2007;107:1228-30.

6. Burmeister HP, Baltzer PA, Dietzel M, et al. Identification of the nervus intermedius using 3T MR imaging. AJNR Am J Neuroradiol 2011;32:460-4.

7. Rhoton AL Jr, Kobayashi S, Hollinshead WH. Nervus intermedius. J Neurosurg 1968;29:609-8.

8. Holste KG, Hardaway FA, Raslan AM, et al. Pain-free and pain-controlled survival after sectioning the nervus intermedius in nervus intermedius neuralgia: a singleinstitution review. J Neurosurg 2018;131:352-9.

9. Yamamoto Y, Hashikata H, Toda H, et al. Nervus intermedius section to mobilize the anterior inferior cerebellar artery in microvascular decompression surgery for hemifacial spasm: a technical case report. World Neurosurg 2019;122:491-4.

10. Hunt JR. On herpetic inflammations of the geniculate ganglion. A new syndrome and its complications. J Nerv Ment Dis 1907;34:73-96.

11. Frese A, Luttmann RJ, Husstedt IW, et al. Geniculate neuralgia as a manifestation of neuroborreliosis. Headache 2002;42:826-8. 